\title{
Adjacent-segment disease following two-level axial lumbar interbody fusion
}

\author{
${ }^{*}$ Alex P. Michael, MD, ${ }^{1}$ Matthew W. Weber, BS, ${ }^{1}$ Kristin R. Delfino, PhD, ${ }^{2}$ and \\ Venkatanarayanan Ganapathy, MD, FRCSC ${ }^{3}$
}

'Division of Neurosurgery and ${ }^{2}$ Center for Clinical Research, Southern Illinois University School of Medicine, Springfield, Illinois; and ${ }^{3}$ Department of Orthopaedic Surgery, University of Tennessee College of Medicine, Chattanooga, Tennessee

OBJECTIVE While long-term studies have evaluated adjacent-segment disease (ASD) following posterior lumbar spine arthrodesis, no such studies have assessed the incidence and prevalence of ASD following axial lumbar interbody fusion (AxiaLIF). The aim of this study was to estimate the incidence of ASD following AxiaLIF.

METHODS The authors retrospectively reviewed the medical records of 149 patients who underwent two-level index AxiaLIF and had at least 2 years of radiographic and clinical follow-up. ASD and pre- and postoperative lumbar lordosis were evaluated in each patient. ASD was defined as both radiographic and clinically significant disease at a level adjacent to a previous fusion requiring surgical intervention. The mean duration of follow-up was 6.01 years.

RESULTS Twenty (13.4\%) of the 149 patients developed ASD during the data collection period. Kaplan-Meier analysis predicted a disease-free ASD survival rate of $95.3 \%(95 \% \mathrm{Cl} 90.4 \%-97.7 \%)$ at 2 years and $89.1 \%(95 \% \mathrm{Cl} 82.8 \%$ -

$93.2 \%$ ) at 5 years for two-level fusion. A laminectomy adjacent to a fusion site was associated with 5.1 times the relative risk of developing ASD. Furthermore, the ASD group had significantly greater loss of lordosis than the no-ASD group ( $p$ $=0.033$ ).

CONCLUSIONS Following two-level AxiaLIF, the rate of symptomatic ASD warranting either decompression or arthrodesis was found to be $4.7 \%$ at 2 years and $10.9 \%$ at 5 years. Adjacent-segment decompression and postoperative loss of lumbar lordosis predicted future development of ASD. To the authors' knowledge, this is the largest reported cohort of patients to undergo two-level AxiaLIF in the United States.

https://thejns.org/doi/abs/10.3171/2019.2.SPINE18929

KEYWORDS axial lumbar interbody fusion; adjacent-segment disease; lumbar fusion; survivorship analysis; lumbar lordosis

\begin{abstract}
A DJACENT-segment disease (ASD) is a well-recognized long-term consequence of lumbar interbody fusion. $8,12,15,16,22,25,27,30$ The literature has shown that minimally invasive lumbar fusion techniques result in similar clinical outcomes as open approaches and offer the advantages of a smaller incision, less soft-tissue trauma, and quicker return to normal activities. ${ }^{31}$ Compared to other minimally invasive options, the axial lumbar interbody fusion (AxiaLIF) system (TranS1 Inc.) has the added benefit of avoiding the critical anterior neurovascular and posterior musculoligamentous structures by utilizing a presacral approach.
\end{abstract}

Despite these proposed advantages of AxiaLIF and its low rates of perioperative morbidity, ${ }^{11,17}$ some have expressed concern regarding long-term outcomes due to the high rates of pseudarthrosis and postoperative loss of lumbar lordosis. ${ }^{13,19}$ Furthermore, risk factors for the development of ASD are not well established. A recent literature review found loss of sagittal balance and adjacent-segment decompression to be the most reliable predictors of ASD. ${ }^{26}$

While long-term studies have evaluated ASD in posterior lumbar spine arthrodesis, no such studies have analyzed ASD following the AxiaLIF technique. In this study, we performed a retrospective investigation to determine

ABBREVIATIONS ASD = adjacent-segment disease; AxiaLIF = axial lumbar interbody fusion. SUBMITTED July 29, 2018. ACCEPTED February 4, 2019.

INCLUDE WHEN CITING Published online April 19, 2019; DOI: 10.3171/2019.2.SPINE18929.

${ }^{*}$ A.P.M. and M.W.W. contributed equally to this work and share first authorship. 
the incidence and prevalence of and risk factors for ASD using a large cohort of patients who have undergone firsttime, two-level interbody fusion through the presacral approach using the AxiaLIF system with concurrent percutaneous pedicle screw fixation. To our knowledge, this study is the largest reported number of two-level AxiaLIF cases in the United States.

\section{Methods}

The institutional review board at Southern Illinois University School of Medicine approved this research project. We believe that all aspects of this research, summarized in this manuscript, were conducted in accordance with American Psychological Association ethical standards.

We retrospectively reviewed the medical records of 165 patients who had had undergone first-time, two-level AxiaLIF. The hospital records, office charts, and radiographs were reviewed by two independent observers (A.P.M., M.W.W.) to determine demographic characteristics, symptoms, preoperative diagnosis, and surgical interventions before and after arthrodesis. All patients had a two-level AxiaLIF performed by a single surgeon at a single academic institution. Of these patients, 11 had posterior lumbar arthrodesis prior to AxiaLIF and were excluded from the study. Of the remaining 154 patients, 5 patients did not have 2-year radiographic and clinical follow-up and were excluded from further analysis (Fig. 1).

The final study group consisted of 149 patients (82 female, 67 male). The mean patient age at the time of the index procedure was 51.45 years (range 22-82 years). All patients had a clinical visit with documentation of function at least 2 years after the index procedure. The mean duration of follow-up was $6.01 \pm 1.00$ years (range 2.83-7.93 years). None of these patients had an acute fracture, spinal

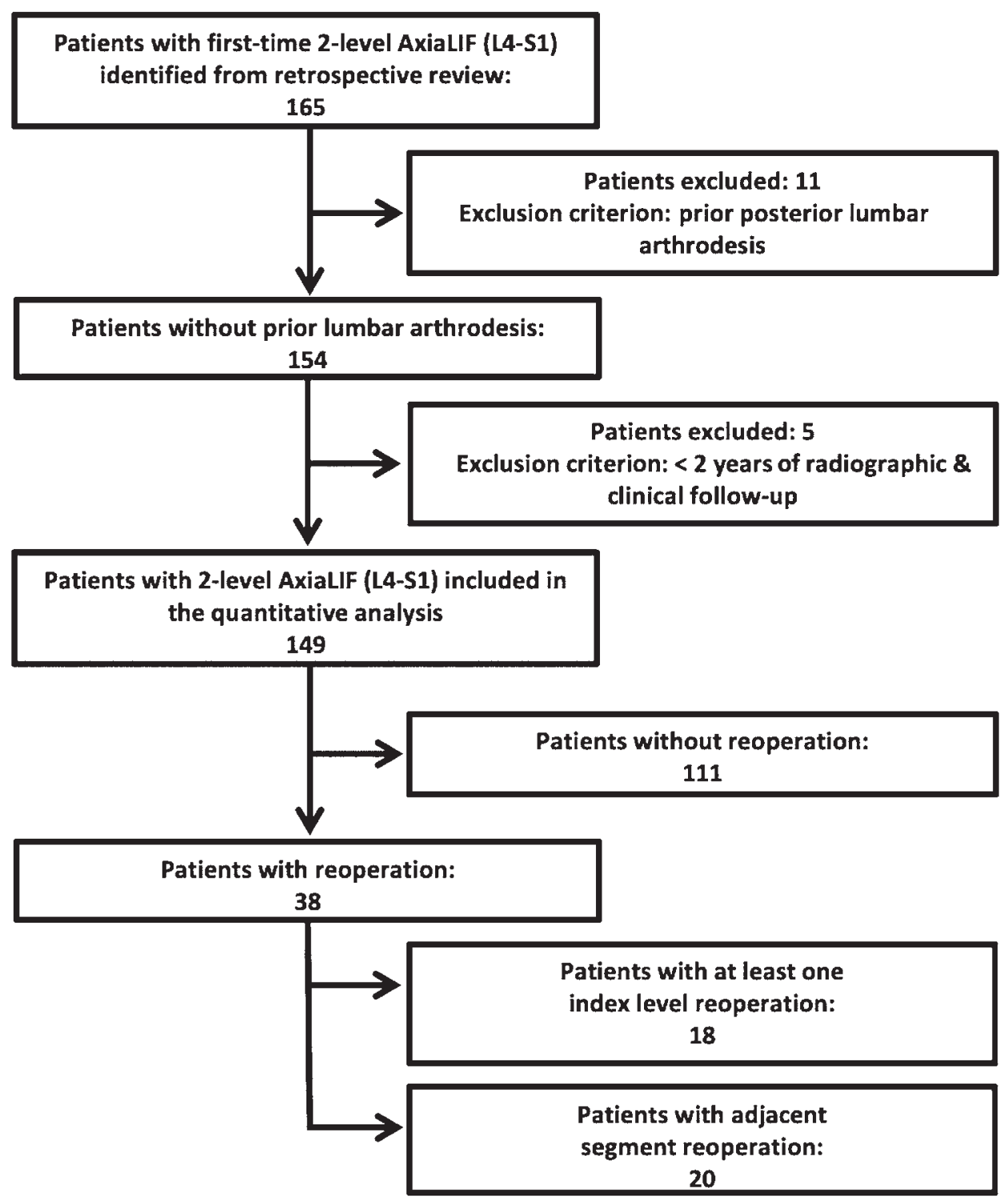

FIG. 1. Flowchart demonstrating patient selection criteria for inclusion in survival analysis. 
neoplasm, or spinal vascular malformation. The primary indications for the index AxiaLIF included spondylolisthesis, dynamic instability ( $>3 \mathrm{~mm}$ on flexion-extension radiographs), or degenerative lumbar scoliosis leading to a neurogenic claudication and/or radiculopathy. ${ }^{9}$ In all patients conservative nonsurgical treatment failed prior to their undergoing surgery.

All patients underwent AxiaLIF via a presacral approach, as per standard technique, followed by percutaneous pedicle screw fixation, as previously described. $7,18,20$ Crushed cancellous allograft bone was mixed with bone marrow aspirate collected during the procedure and used as graft material.

ASD was defined as both radiographic and clinically significant disease at a level adjacent to a previous fusion requiring surgical intervention. ${ }^{2,45}$ For the purposes of this study, the adjacent segment was considered to be the L3 vertebra for the two-level (L4-S1) AxiaLIF.

Two independent observers blinded to surgical outcome retrospectively assessed patients' lumbar lordosis using standing lateral plain radiographs. Pre-AxiaLIF radiographic measurements were assessed nearest to the procedure date, and post-AxiaLIF measurements were assessed just prior to reoperation in the ASD group or at the most recent follow-up in the no-ASD group. All patients underwent standing lateral lumbar radiography both preand postoperatively; however, not all preoperative radiographs were available to view at the time of this study. Additionally, some radiographs were of insufficient quality to accurately assess lumbar lordosis and were excluded (Fig. 2). Lumbar lordosis was defined as the Cobb angle formed by the cranial endplate of L1 and the cranial endplate of S1. Ten $(50 \%)$ of the 20 patients with ASD and $64(57.7 \%)$ of the 111 patients without any reoperation had both preand post-AxiaLIF radiographs and were included in this analysis.

\section{Statistical Analysis}

Kaplan-Meier survivorship analysis was used to assess disease-free survival of index and adjacent-segment disease. The annual incidence and prevalence were de-

Patients with first-time 2-level AxiaLIF (L4-S1)

identified from retrospective review:

149

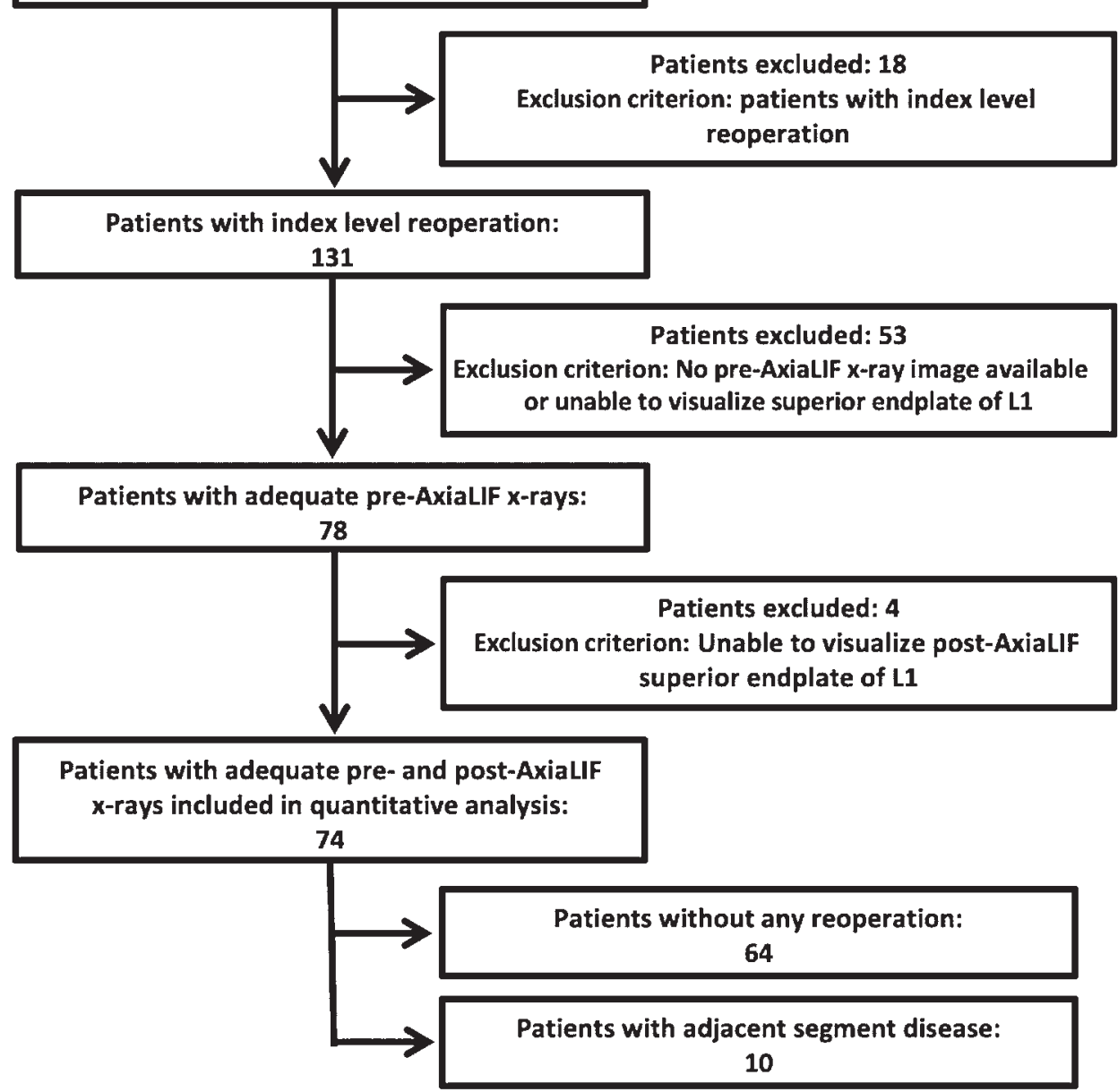

FIG. 2. Flowchart demonstrating patient selection criteria for inclusion in radiographic analysis of lumbar lordosis. 
termined for each year of follow-up. Student t-tests and chi-square tests were used for continuous and categorical variables, respectively. Binary logistic regression analysis was used to determine the relationship between lumbar lordosis and ASD. Additionally, interclass correlation coefficient and mean absolute differences between the two independent lumbar lordosis measurements were calculated. Finally, we assessed the relative risk of developing ASD after having an adjacent-segment decompression prior to the AxiaLIF. Statistical analyses were performed with SAS/STAT software version 14.3 (SAS Institute Inc.). Where appropriate, data are expressed as a mean with $95 \%$ CI. Significance was set at $\mathrm{p}<0.05$.

\section{Results}

Thirty-eight (25.5\%) of the 149 patients had an additional lumbar surgery during the study period. Twenty patients (13.4\%) were found to have a reoperation at the adjacent L3 segment and were considered as having ASD. The primary reasons for reoperation at the adjacent level are summarized in Table 1. Three patients (15\%) had foraminal stenosis, 5 patients $(25 \%)$ had both central and foraminal stenosis, 6 patients (30\%) had spondylolisthesis, and 6 patients (30\%) had kyphoscoliosis. Eighteen patients (12.1\%) underwent a total of 31 index-level reoperations. The majority of patients who underwent reoperation at an index level had persistent foraminal or central stenosis. Six $(4.0 \%)$ of the 149 patients developed a symptomatic pseudarthrosis requiring a reoperation. Pseudarthrosis was based on lack of union on CT scans and/or motion of the construct at the level of attempted fusion.

There was no significant difference between ASD and non-ASD groups with regard to sex $(\mathrm{p}=0.631)$ or age $(\mathrm{p}=$ 0.255 ). Of the 149 patients, 7 had previously undergone a laminectomy at the adjacent L3 level prior to the two-level AxiaLIF. Four of these 7 patients developed ASD, while 16 of the 126 without pre-AxiaLIF adjacent decompression developed ASD (Table 2). Patients with an adjacentsegment decompression prior to AxiaLIF were 5.1 times more likely to develop ASD than those without adjacentsegment decompression (95\% CI 2.3-11.2; $\mathrm{p}=0.007)$.

Kaplan-Meier survivorship analysis was performed to

TABLE 1. Diagnosis for index- or adjacent-level reoperation

\begin{tabular}{cc}
\hline \multicolumn{1}{c}{ Diagnosis } & Incidence \\
\hline Index-level reoperation & \\
\hline Foraminal stenosis & $18(58 \%)$ \\
\hline Central \& foraminal stenosis & $5(16 \%)$ \\
\hline Spondylolisthesis & $1(3 \%)$ \\
\hline Kyphoscoliosis & $1(3 \%)$ \\
\hline Pseudarthrosis & $6(19 \%)$ \\
\hline Adjacent-level reoperation & $3(15 \%)$ \\
\hline Foraminal stenosis & $5(25 \%)$ \\
\hline Central \& foraminal stenosis & $6(30 \%)$ \\
\hline Spondylolisthesis & $6(30 \%)$ \\
\hline Kyphoscoliosis &
\end{tabular}

TABLE 2. Pre-AxiaLIF adjacent-segment decompression versus ASD

\begin{tabular}{lrc}
\hline Condition & ASD & No ASD \\
\hline Pre-AxiaLIF adjacent decompression at L3 & 4 & 3 \\
\hline No pre-AxiaLIF adjacent decompression at L3 & 16 & 126 \\
\hline
\end{tabular}

assess the rate of disease-free survival for patients with a two-level (L4-S1) fusion. ASD-free survival was estimated to be $95.3 \%(95 \%$ CI $90.4 \%-97.7 \%)$ at 2 years and $89.1 \%$ (95\% CI 82.8\%-93.2\%) at 5 years. The estimated disease-free survival rate for any reoperation at either the index or adjacent levels was $83.2 \%$ (95\% CI 76.2\%-88.3\%) at 2 years and $75.6 \%$ (95\% CI $67.8 \%-81.8 \%$ ) at 5 years (Fig. 3). The mean annual incidence of ASD was $2.19 \%$ (95\% CI $1.16 \%-3.22 \%$; range $0 \%-4.27 \%$ ). The results of the Kaplan-Meier analysis for ASD are shown in Table 3. Kaplan-Meier analysis for any reoperation is shown in Table 4.

Radiographic measurement of pre- and post-AxiaLIF lumbar lordosis was conducted in the ASD group and the no-ASD group by two independent observers. There was high interobserver reliability for lumbar lordosis calculations (interclass correlation coefficient $0.97,95 \%$ CI 0.96-0.98), and a mean absolute difference between lumbar lordosis measurements in a subject was found to be 1.98. Intragroup comparison of lumbar lordosis revealed a significant loss of lordosis post-AxiaLIF compared to preAxiaLIF in both the ASD $\left(45.9^{\circ}\right.$ vs $\left.54.9^{\circ}, \mathrm{p}=0.001\right)$ and no-ASD $\left(48.0^{\circ}\right.$ vs $\left.51.12^{\circ}, \mathrm{p}=0.004\right)$ groups. Intergroup lumbar lordosis analysis showed a significantly greater loss of lordosis post-AxiaLIF in the ASD group than in the no-ASD group $\left(-9.0^{\circ}\right.$ vs $\left.-3.1^{\circ}, \mathrm{p}=0.033\right)$. Binary logistic regression revealed a greater loss of post-AxiaLIF lumbar lordosis predicted future development of ASD (OR 1.11, 95\% CI 1.01-1.22; $\mathrm{p}<0.039$ ).

\section{Discussion}

The exact pathogenesis of ASD is unknown. Several studies suggest that lumbar arthrodesis may increase biomechanical stress with physiological loading at the level adjacent to the fusion, accelerating degenerative disc disease and creating instability. ${ }^{12,34}$ It has also been proposed that loss of the posterior tension band function may add to adjacent-segment instability and accelerate disc degeneration. ${ }^{6}$ The proposed advantage of minimally invasive approaches in the lumbar spine is preservation of the paraspinal musculature and ligamentous structures to prevent iatrogenic causes of adjacent-segment degeneration. ${ }^{26,32,35}$ However, it has been shown that minimally invasive percutaneous pedicle screw placement may result in a higher rate of facet violation, which may lead to adjacent-segment instability. ${ }^{24}$

The AxiaLIF procedure has been shown to have a low rate of perioperative complications in post-marketing surveillance analyses, ${ }^{11,17}$ though some authors have expressed concern regarding long-term biomechanical stability. Postoperative radiographic evaluation within 1 week of the AxiaLIF procedure has revealed a transient 


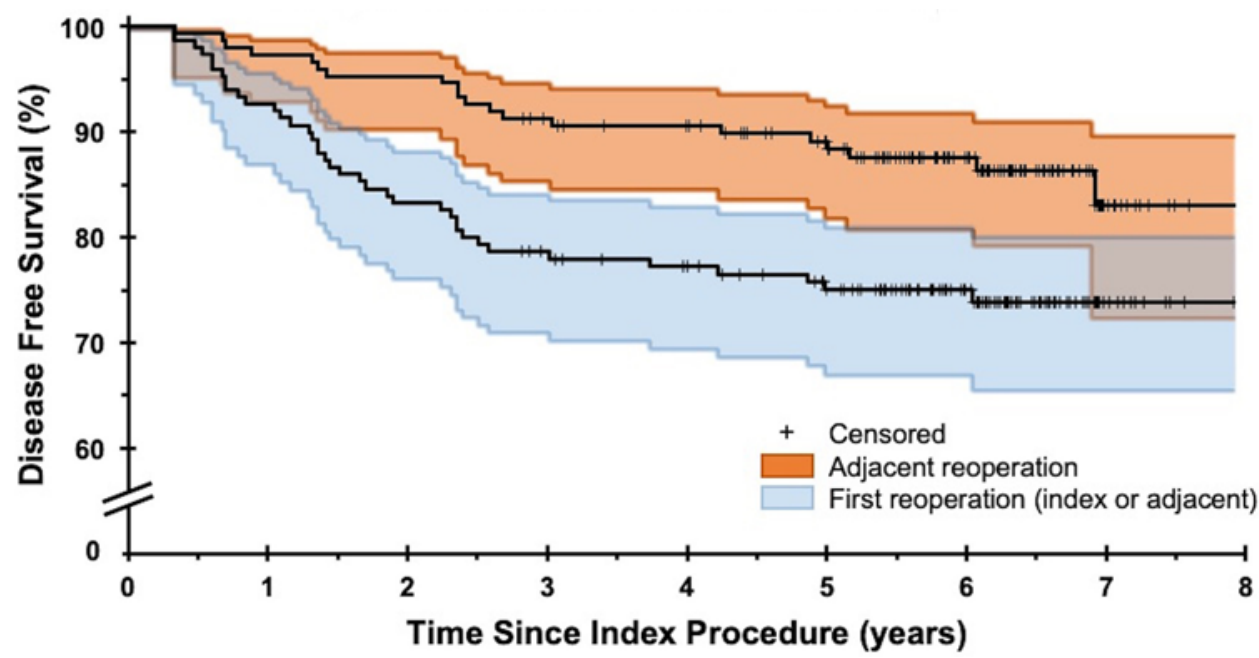

FIG. 3. Kaplan-Meier survivorship curves showing the predicted percentage of patients remaining disease free (without reoperation). Shaded areas represent the $95 \% \mathrm{Cls}$. Censored patients are denoted by crosses. Survivorship curve representing time until adjacent-level reoperation is denoted with red shading, while time until first reoperation (either index or adjacent) is denoted with blue shading. Figure is available in color online only.

increase in segmental lumbar lordosis and disc space distraction. ${ }^{13,19}$ However, at follow-up imaging 2 years later, disc height and lumbar lordosis decreased to less than preoperative values. Furthermore, there have been reports of high rates of nonunion ${ }^{13,17,19}$ manifesting as radiolucency around the transsacral rod and subsequent decrease of construct stiffness.

Here we present the largest cohort analysis of patients who underwent a two-level AxiaLIF. The predicted prevalence of symptomatic and radiographic degeneration at an adjacent segment warranting either decompression or arthrodesis following two-level AxiaLIF was found to be $10.9 \%$ at 5 years. Retrospective studies by Ghiselli et al. ${ }^{8}$ and Sears et al. ${ }^{28}$ both used Kaplan-Meier survivorship analysis and found ASD requiring reoperation after posterior lumbar arthrodesis to be $16.5 \%$ and $13.6 \%$, respectively, at 5 years. They also reported the mean annual incidence of ASD to be $3.9 \%$ and $2.5 \%$, respectively, compared to $2.19 \%$ in our study. These retrospective studies included all lumbar arthrodesis regardless of vertebral level or length of the construct. To our knowledge, there have been no studies evaluating ASD following posterior lumbar arthrodesis only at L4-S1 using survivorship analysis for direct comparison.

In the current study, fusion of the lumbar spine using the AxiaLIF technique resulted in significant postoperative loss of lumbar lordosis in both ASD and no-ASD groups. This may be due to an inherent flaw in the technique in which the threads of the transsacral rod may be inadequate to maintain long-term spinal column support without a solid interposition graft..$^{13}$ The result may be subsidence of the AxiaLIF rod and loss of lumbar lordosis. ${ }^{3,13,19}$

Poor sagittal alignment can have deleterious effects on spinal segments adjacent to a fusion. Postsurgical loss of lumbar lordosis has been found to cause hypermobility $^{1}$ and increased biomechanical stress ${ }^{23,29}$ at the adjacent segment. The rigid, nonphysiological kyphotic fusion of the spine may induce abnormal loading and increased motion at the adjacent mobile segment as the patient attempts to restore range of motion. In our study, we found a significantly greater loss of lumbar lordosis in the ASD

TABLE 3. Kaplan-Meier survivorship life table for ASD

\begin{tabular}{cccccccccc}
\hline & & & \multicolumn{1}{c}{ Annual } \\
Yrs of FU & No. Entered & No. Censored & No. at Risk & Terminal Events & Incidence (\%) & DFS (\%) & SE (\%) & Prevalence (\%) \\
\hline 1 & 149 & 0 & 149 & 4 & 2.7 & 97.3 & 1.3 & 2.7 \\
\hline 2 & 145 & 0 & 145 & 3 & 2.1 & 95.3 & 1.7 & 4.7 \\
\hline 3 & 142 & 3 & 140.5 & 6 & 4.3 & 91.2 & 2.3 & 8.8 \\
\hline 4 & 133 & 4 & 131 & 1 & 0.8 & 90.5 & 2.4 & 9.5 \\
\hline 5 & 128 & 10 & 123 & 2 & 1.6 & 89.1 & 2.6 & 10.9 \\
\hline 6 & 116 & 35 & 98.5 & 2 & 2 & 87.3 & 2.8 & 12.7 \\
\hline 7 & 79 & 60 & 49 & 2 & 4.1 & 83.7 & 3.7 & 16.3 \\
\hline 8 & 17 & 17 & 8.5 & 0 & 0 & 83.7 & 3.7 & 16.3 \\
\hline
\end{tabular}

DFS = disease-free survival; FU = follow-up; SE = standard error. 
TABLE 4. Kaplan-Meier survivorship life table for first reoperation (adjacent segment or index level)

\begin{tabular}{ccccccccc}
\hline Yrs of FU & No. Entered & No. Censored & No. at Risk & Terminal Events & $\begin{array}{c}\text { Annual } \\
\text { Incidence (\%) }\end{array}$ & DFS (\%) & SE (\%) & Prevalence (\%) \\
\hline 1 & 149 & 0 & 149 & 11 & 7.4 & 92.6 & 2.1 & 7.4 \\
\hline 2 & 138 & 0 & 138 & 14 & 10.1 & 83.2 & 2.4 & 16.8 \\
\hline 3 & 124 & 3 & 122.5 & 7 & 5.7 & 78.5 & 1.8 & 21.5 \\
\hline 4 & 114 & 4 & 112 & 2 & 1.8 & 77.1 & 1.0 & 22.9 \\
\hline 5 & 108 & 7 & 104.5 & 2 & 1.9 & 75.6 & 1.0 & 24.4 \\
\hline 6 & 99 & 32 & 83 & 1 & 1.2 & 74.7 & 0.9 & 25.3 \\
\hline 7 & 66 & 52 & 40 & 1 & 2.5 & 72.8 & 1.8 & 27.2 \\
\hline 8 & 13 & 13 & 6.5 & 0 & 0 & 72.8 & 0 & 27.2 \\
\hline
\end{tabular}

group than the no-ASD group, and a greater loss of lumbar lordosis may predispose a patient to the development of ASD. Other studies have also reported similar results., ${ }^{5,21}$ Together, these findings suggest that failure to maintain physiological sagittal balance may be an important risk factor for the development of ASD. However, the causality is not established in this study, and it is possible that the ASD leads to further loss of lordosis.

Other fusion techniques such as posterior lumbar fusion and transforaminal lumbar interbody fusion may allow better endplate preparation and provide a larger surface area for interbody fusion to occur. They may allow for lordotic spacers to maintain natural sagittal alignment. The AxiaLIF technique does not afford lordosis correction. Most of the lordotic curvature in the lumbar spine is maintained across L4-S1 and is therefore especially important at these critical levels.

During the study data collection period, we found that 6 (4.0\%) of the 149 patients developed a symptomatic pseudarthrosis requiring a reoperation. Prior studies on twolevel AxiaLIF have reported similar pseudarthrosis rates, though they are limited by a small patient cohort. ${ }^{13,17}$

The adjacent level was considered to be L3 for the two-level (L4-S1) AxiaLIF performed in this study. We found that an adjacent-segment spinal decompression was associated with developing ASD. Other studies have also reported spinal decompression adjacent to a fusion as a significant risk factor for the development of ASD; ${ }^{6,28}$ however, another study found having an intact lamina adjacent to a spinal fusion to be a significant risk factor for the development of ASD. ${ }^{2}$ Such variance in reported results could be attributed to difference in follow-up time, operative location in the lumbar spine, number of segments fused, sample size, and operative technique.

Whether longer-length fusions predispose patients to ASD remains controversial in the literature. Several authors have found longer-length fusions to be associated with increased incidence of ASD, ${ }^{2,10,28}$ while another group found that single-level fusions were more likely to have ASD than multilevel fusions. ${ }^{8}$ Others have reported no significant difference between fusion length and incidence of ASD. ${ }^{14,33}$ We did not have a matched group of patients who had undergone a single-level AxiaLIF for comparison. A definitive determination of the impact of fusion length on ASD requires further study.

\section{Limitations}

Our study had several important limitations. First, the study was a retrospective review of a single surgeon's experience and has an inherent selection bias. Therefore, patients may have been lost to follow-up if they transitioned out of the practice. The study also includes a heterogeneous patient population. Although the Student t-test and chi-square test were performed to assess the contributions of independent variables such as age and sex, accounting for other potential cofounders may have provided a stronger correlation between independent variables and ASD. Because this was a retrospective and radiographic study, we did not assess pre- and postoperative clinical outcomes measures. Had this information been available, we would have more reliably been able to assess independent risk factors. Finally, our study lacked a control group for comparison. A matched cohort of patients who elected to forgo the index arthrodesis would have provided an ideal control group to assess for further degenerative changes at an adjacent segment and help determine if ASD is an iatrogenic result of the arthrodesis itself or a de novo manifestation of the patient's natural propensity for lumbar degeneration.

\section{Conclusions}

To our knowledge, this study is the first to estimate the rate of ASD following two-level AxiaLIF using survivorship analysis. We found the rate of symptomatic ASD warranting surgical intervention to be $4.7 \%$ at 2 years and $10.9 \%$ at 5 years. An adjacent-segment decompression prior to AxiaLIF was associated with 5.1 times the relative risk of developing ASD. Furthermore, after the index AxiaLIF procedure, patients who had a greater loss of lumbar lordosis were at greater risk for developing ASD than those who did not. There appears to be significant heterogeneity of reported ASD risk factors in the literature; therefore, prospective trials are warranted for further evaluation.

\section{References}

1. Akamaru T, Kawahara N, Tim Yoon S, Minamide A, Su Kim $\mathrm{K}$, Tomita K, et al: Adjacent segment motion after a simulated lumbar fusion in different sagittal alignments: a biomechanical analysis. Spine (Phila Pa 1976) 28:1560-1566, 2003

2. Alentado VJ, Lubelski D, Healy AT, Orr RD, Steinmetz MP, 
Benzel EC, et al: Predisposing characteristics of adjacent segment disease after lumbar fusion. Spine (Phila Pa 1976) 41:1167-1172, 2016

3. Aryan HE, Newman CB, Gold JJ, Acosta FL Jr, Coover C, Ames CP: Percutaneous axial lumbar interbody fusion (AxiaLIF) of the L5-S1 segment: initial clinical and radiographic experience. Minim Invasive Neurosurg 51:225-230, 2008

4. Bydon M, Macki M, Kerezoudis P, Sciubba DM, Wolinsky JP, Witham TF, et al: The incidence of adjacent segment disease after lumbar discectomy: a study of 751 patients. J Clin Neurosci 35:42-46, 2017

5. Djurasovic MO, Carreon LY, Glassman SD, Dimar JR II, Puno RM, Johnson JR: Sagittal alignment as a risk factor for adjacent level degeneration: a case-control study. Orthopedics 31:546, 2008

6. Ekman P, Möller H, Hedlund R: Predictive factors for the outcome of fusion in adult isthmic spondylolisthesis. Spine (Phila Pa 1976) 34:1204-1210, 2009

7. Erkan S, Wu C, Mehbod AA, Hsu B, Pahl DW, Transfeldt EE: Biomechanical evaluation of a new AxiaLIF technique for two-level lumbar fusion. Eur Spine J 18:807-814, 2009

8. Ghiselli G, Wang JC, Bhatia NN, Hsu WK, Dawson EG: Adjacent segment degeneration in the lumbar spine. J Bone Joint Surg Am 86-A:1497-1503, 2004

9. Ghogawala Z, Benzel EC, Amin-Hanjani S, Barker FG II, Harrington JF, Magge SN, et al: Prospective outcomes evaluation after decompression with or without instrumented fusion for lumbar stenosis and degenerative Grade I spondylolisthesis. J Neurosurg Spine 1:267-272, 2004

10. Gillet P: The fate of the adjacent motion segments after lumbar fusion. J Spinal Disord Tech 16:338-345, 2003

11. Gundanna MI, Miller LE, Block JE: Complications with axial presacral lumbar interbody fusion: a 5-year postmarketing surveillance experience. SAS J 5:90-94, 2011

12. Hilibrand AS, Robbins M: Adjacent segment degeneration and adjacent segment disease: the consequences of spinal fusion? Spine J 4 (6 Suppl):190S-194S, 2004

13. Hofstetter CP, Shin B, Tsiouris AJ, Elowitz E, Härtl R: Radiographic and clinical outcome after 1- and 2-level transsacral axial interbody fusion: clinical article. J Neurosurg Spine 19:454-463, 2013

14. Kumar MN, Jacquot F, Hall H: Long-term follow-up of functional outcomes and radiographic changes at adjacent levels following lumbar spine fusion for degenerative disc disease. Eur Spine J 10:309-313, 2001

15. Lee JC, Lee SH, Peters C, Riew KD: Adjacent segment pathology requiring reoperation after anterior cervical arthrodesis: the influence of smoking, sex, and number of operated levels. Spine (Phila Pa 1976) 40:E571-E577, 2015

16. Lehmann TR, Spratt KF, Tozzi JE, Weinstein JN, Reinarz SJ, el-Khoury GY, et al: Long-term follow-up of lower lumbar fusion patients. Spine (Phila Pa 1976) 12:97-104, 1987

17. Lindley EM, McCullough MA, Burger EL, Brown CW, Patel VV: Complications of axial lumbar interbody fusion. J Neurosurg Spine 15:273-279, 2011

18. Luther N, Tomasino A, Parikh K, Härtl R: Neuronavigation in the minimally invasive presacral approach for lumbosacral fusion. Minim Invasive Neurosurg 52:196-200, 2009

19. Marchi L, Oliveira L, Coutinho E, Pimenta L: Results and complications after 2-level axial lumbar interbody fusion with a minimum 2-year follow-up. J Neurosurg Spine 17:187-192, 2012

20. Marotta N, Cosar M, Pimenta L, Khoo LT: A novel minimally invasive presacral approach and instrumentation technique for anterior L5-S1 intervertebral discectomy and fusion: technical description and case presentations. Neurosurg Focus 20(1):E9, 2006

21. Maysumoto TO: Spinopelvic sagittal imbalance as a risk factor for adjacent-segment disease after single-segment posteri- or lumbar interbody fusion. J Neurosurg Spine 26:435-440, 2017

22. Miyakoshi N, Abe E, Shimada Y, Okuyama K, Suzuki T, Sato K: Outcome of one-level posterior lumbar interbody fusion for spondylolisthesis and postoperative intervertebral disc degeneration adjacent to the fusion. Spine (Phila Pa 1976) 25:1837-1842, 2000

23. Oda I, Cunningham BW, Buckley RA, Goebel MJ, Haggerty CJ, Orbegoso CM, et al: Does spinal kyphotic deformity influence the biomechanical characteristics of the adjacent motion segments? An in vivo animal model. Spine (Phila Pa 1976) 24:2139-2146, 1999

24. Patel RD, Graziano GP, Vanderhave KL, Patel AA, Gerling MC: Facet violation with the placement of percutaneous pedicle screws. Spine (Phila Pa 1976) 36:E1749-E1752, 2011

25. Penta M, Sandhu A, Fraser RD: Magnetic resonance imaging assessment of disc degeneration 10 years after anterior lumbar interbody fusion. Spine (Phila Pa 1976) 20:743-747, 1995

26. Radcliff KE, Kepler CK, Jakoi A, Sidhu GS, Rihn J, Vaccaro $A R$, et al: Adjacent segment disease in the lumbar spine following different treatment interventions. Spine J 13:13391349,2013

27. Schlegel JD, Smith JA, Schleusener RL: Lumbar motion segment pathology adjacent to thoracolumbar, lumbar, and lumbosacral fusions. Spine (Phila Pa 1976) 21:970-981, 1996

28. Sears WR, Sergides IG, Kazemi N, Smith M, White GJ, Osburg B: Incidence and prevalence of surgery at segments adjacent to a previous posterior lumbar arthrodesis. Spine $\mathbf{J}$ 11:11-20, 2011

29. Umehara S, Zindrick MR, Patwardhan AG, Havey RM, Vrbos LA, Knight GW, et al: The biomechanical effect of postoperative hypolordosis in instrumented lumbar fusion on instrumented and adjacent spinal segments. Spine (Phila Pa 1976) 25:1617-1624, 2000

30. Wai EK, Santos ER, Morcom RA, Fraser RD: Magnetic resonance imaging 20 years after anterior lumbar interbody fusion. Spine (Phila Pa 1976) 31:1952-1956, 2006

31. Wang J, Zhou Y, Zhang ZF, Li CQ, Zheng WJ, Liu J: Comparison of one-level minimally invasive and open transforaminal lumbar interbody fusion in degenerative and isthmic spondylolisthesis grades 1 and 2. Eur Spine J 19:1780-1784, 2010

32. Watanabe K, Matsumoto M, Ikegami T, Nishiwaki Y, Tsuji T, Ishii K, et al: Reduced postoperative wound pain after lumbar spinous process-splitting laminectomy for lumbar canal stenosis: a randomized controlled study. J Neurosurg Spine 14:51-58, 2011

33. Wiltse LL, Radecki SE, Biel HM, DiMartino PP, Oas RA, Farjalla G, et al: Comparative study of the incidence and severity of degenerative change in the transition zones after instrumented versus noninstrumented fusions of the lumbar spine. J Spinal Disord 12:27-33, 1999

34. Xia XP, Chen HL, Cheng HB: Prevalence of adjacent segment degeneration after spine surgery: a systematic review and meta-analysis. Spine (Phila Pa 1976) 38:597-608, 2013

35. Yee TJ, Terman SW, La Marca F, Park P: Comparison of adjacent segment disease after minimally invasive or open transforaminal lumbar interbody fusion. J Clin Neurosci 21:1796-1801, 2014

\section{Disclosures}

The authors report no conflict of interest concerning the materials or methods used in this study or the findings specified in this paper.

\section{Author Contributions}

Conception and design: Michael, Ganapathy. Acquisition of data: 
Michael, Weber, Ganapathy. Analysis and interpretation of data: Michael, Ganapathy. Drafting the article: Michael, Weber. Critically revising the article: all authors. Reviewed submitted version of manuscript: all authors. Approved the final version of the manuscript on behalf of all authors: Michael. Statistical analysis: Delfino. Administrative/technical/material support: Ganapathy. Study supervision: Ganapathy.

\section{Supplemental Information}

\section{Previous Presentations}

A preliminary abstract was presented in poster form at the Congress of Neurological Surgeons national meeting in Boston, MA, October 2017.

\section{Correspondence}

Alex P. Michael: Southern Illinois University School of Medicine, Springfield, IL.amichael@siumed.edu. 